\title{
Die glazifluvialen Sedimente im unteren Günztal (Bayerisch Schwaben/Deutschland) nach morpho- und pedostratigraphischen Befunden sowie TL-Daten
}

\author{
Stefan Miara \& KonRad RÖGnER*)
}

Pleistocene, Mindel, Riss, Glaciofluvial deposits, TL-dating, Aeolian cover sediment, Günz valley, Bayrisch Schwaben/Germany

\begin{abstract}
Kurzfassung: Bisher wurden die glazifluvialen Schotter im unteren Günztal als eine einheitliche Akkumulation aufgefaßt. Deckschichten- und schotterstratigraphische Untersuchungen führten - in Verbindung mit TL-Datierungen zu einer Differenzierung, die besagt, daß neben den Schottern der oberen Hochterrasse nun auch mindeleiszeitliche Sedimente nachgewiesen werden können. Hinweise auf letztere sind die im Vergleich zu den Hochterrassenschottern wesentlich höher liegenden Schotterbasiswerte. Zusätzlich kann die Schotterbasis des mindeleiszeitlichen Schotters mit derjenigen des Grönenbach-Schwaighausener Schotters (PENCKs mindeleiszeitlicher Schotter der „vier Felder um Memmingen“) im Süden der Iller-Lech-Platte verbunden werden.

Auch die pedostratigraphische Gliederung der jeweiligen Lößdeckschichten in Autenried und Günzburg weist auf ein unterschiedliches Alter der beiden Schotterkörper hin. Bei beiden glazifluvialen Sedimentkörpern handelt es sich nach den vorgestellten Ergebnissen nicht um Teilfelder (ein und derselben Vergletscherung), sondern um Hauptfelder verschieden alter "glazialer Serien".
\end{abstract}

[The glaciofluvial sediments in the lower valley of the river "Günz" (Bavarian Swabia/Germany) as a result of morphostratigraphic and pedostratigraphic investigations and TL-Ages]

\begin{abstract}
Until now in the lower valley of the river "Günz" the glaciofluvial gravel deposits were interpreted as an accumulation of the same glaciation (Riss). But the result of the stratigraphic investigation of loess cover sediments and glaciofluvial gravel layers - in connection with TL (Thermoluminescence)-datings - show that a mindelian gravel body lies beside the gravel deposits of the rissian upper high-terrace. The comparison of both subfaces of the gravel layers demonstrate that the older mindelian gravel deposits can be found on a higher level.

Moreover, it is possible to connect the subface of the mindelian gravel with the subface of the "GrönenbachSchwaighausener" gravel (it is the mindelian gravel of A. PENCK's classical subdivision of the Ice Age with four glaciations in the area of Memmingen) in the south of the "Iller-Lech"-region.
\end{abstract}

*) Anschriften der Verfasser: Prof. Dr. K. RÖGNER und Dipl--Geogr. S. Miara, Ludwig-Maximilians-Universität München, Institut für Geographie, Luisenstr. 37, 80333 München
Furthermore, the stratigraphic division of the aeolian cover sediments at the localities of "Autenried" and "Günzburg" indicates the different ages of both gravel terraces (rissian and mindelian age).

In that case the development of both gravel terraces do not belong to the same glaciation; they represent the main gravel deposits of two different glaciation periods.

\section{Einleitung}

Die oberen Hochterrassenschotter (oHT) im unteren Günztal (zwischen Günzburg und Tafertshofen, Abb. 1) waren in der Vergangenheit schon mehrmals Gegenstand verschiedener Untersuchungen. Ihre stratigraphische Einordnung weicht jedoch unvertretbar voneinander ab. Denn sie werden einmal als Ablagerung der Rißeiszeit angesehen (PENCK \& BrüCKNer 1901-1909; EBerl 1930; SChaEFer in Graul et al. 1951; Graul 1952, 1953; Sinn 1972; Jerz \& WaGNER 1978; HABbE \& RÖGNER 1989b), während andererseits für ihre Entstehung Schmelzwässer der Mindeleiszeit verantwortlich sein sollen (LÖsCHER 1974, 1976; Löscher et al. 1979). Für beide Ansichten gibt es gewichtige Argumente; schotterstratigraphische für die Rißeiszeit, deckschichtenstratigraphische für die Mindeleiszeit. Diese Diskrepanz war Ansatzpunkt der eigenen Studien. Sie beschränkten sich nicht nur auf das Ermitteln von Schotterbasiswerten (Schotterunterkanten nach Löscher 1974: 67) und auf die daraus abgeleitete Rekonstruktion der fossilen Abflußbahnen, sondern sie schlossen auch eine flächendeckende Kartierung der Deckschichten ein. Als repräsentative Lokalitäten wurden die Deckschichtenprofile in Autenried und Günzburg für weitere Gelände- und Laboruntersuchungen ausgewählt.

Das Einmessen der Schichtgrenzen (Tertiär/Quartär = T/Q-Grenze) erfolgte mit einem barometrischen Höhenmesser der Firma Thommen (Typ 3B4) ausgehend von Höhenfixpunkten. Die Untersuchung der Bodenproben aus den Löß-Deckschichten wurde am Institut für Bodenkunde der Landes-, Lehrund Versuchsanstalt für Landwirtschaft, Weinbau und Gartenbau in Trier von S. MiaRa durchgeführt. Die Thermoluminszenz-Datierungen nahm Dr. ZÖL- 


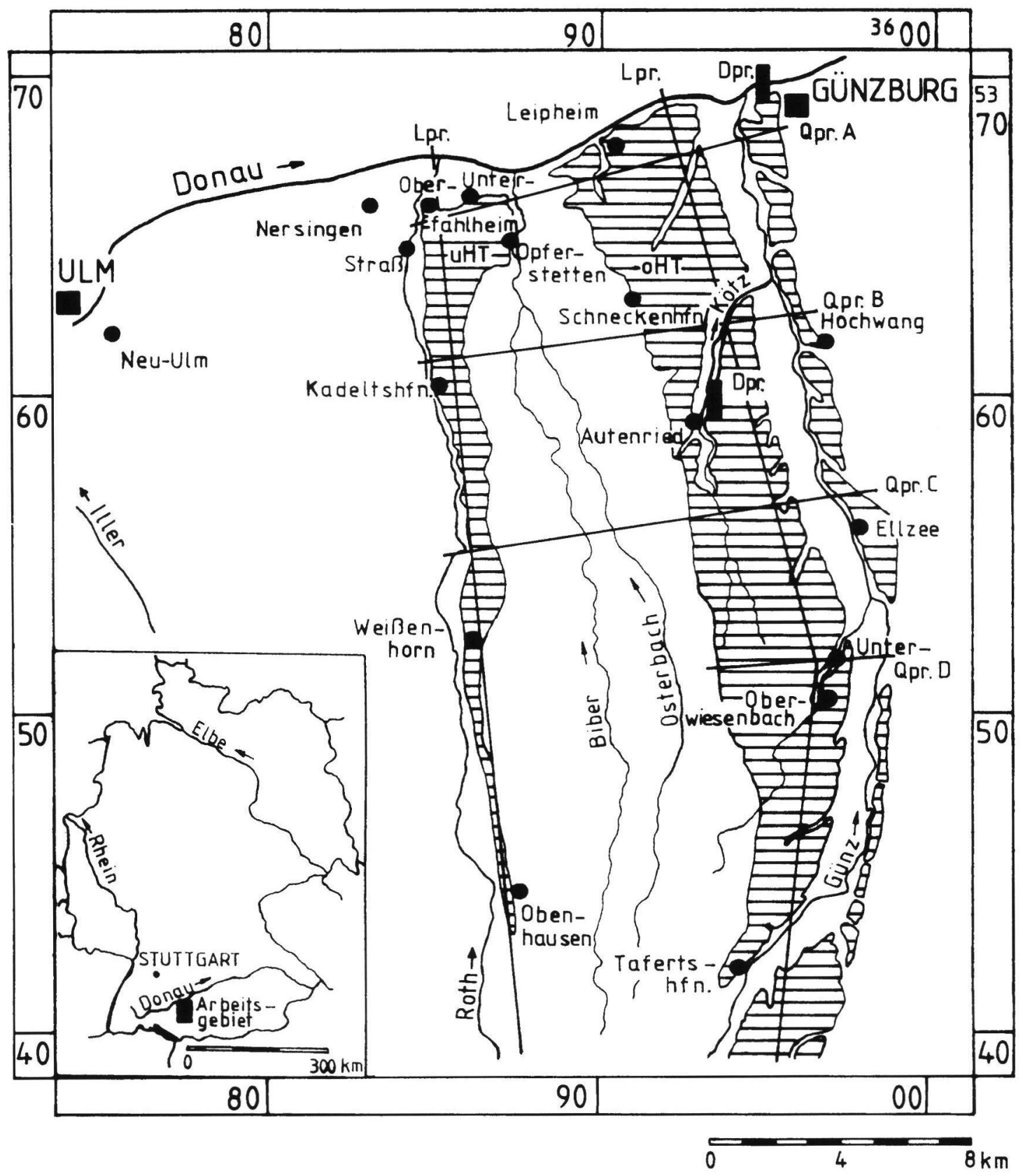

\section{LEGENDE}

Hochterrassenschotter; RiB

mit LöB- u. LöBlehmüberdeckung (ca. $6 \mathrm{~m}$ )

(nach JERZ et al. 1975) 
LER (Max-Planck-Institut für Kernphysik in Heidelberg) vor.

\section{Kurzer forschungsgeschichtlicher Überblick}

Untersuchungen der Schotterfelder von Memmingen $(=$ Vorfeld des pleistozänen Iller-Gletschers) veranlaßten A. PENCK eine Viergliederung des Eiszeitalters vorzunehmen. Jedes der dort treppenartig abgelagerten Schotterfelder (Memminger Feld, Hitzenhofener Feld, Grönenbacher Feld und Hochfeld als Hauptfelder) wurde jeweils einer eigenen Vereisung („Glaziale Serie“) zugeordnet (Würm, Riß, Mindel und Günz). Hochterrassen sind als Bildungen der Rißeiszeit aufzufassen. Ein Blick auf die grundlegenden PENCKschen Querprofile (PENCK \& BRÜCKNER 1901-1909: Abb. 3 und 4) zeigt aber schon, daß es zwei verschieden hoch gelegene Hochterrassen gibt, deren "doppeltes“ Vorkommen mit einer Talverlegung begründet wurde (PENCK \& BRÜCKNER 1901-09: 28-33, 55, 110). EBERl (1930: 108, 263, 266), der die Rißeiszeit in zwei Stadien (Riß I und II) aufgliederte, faßt die Günztal-Hochterrasse ebenfalls rißeiszeitlich auf; sie sei eine Geröllschüttung ausgehend von den älteren R I-Moränen im Dietmannsrieder Zweigbecken.

Auch Schaffer (in Graul, Schaefer \& Weidenbach 1951: 101-103) und Graul (1952: 143-144, 1953: $26-$ 28) ließen am rißeiszeitlichen Alter der Hochterrasse im Günztal (von SchAEFER in Graul et al. [1951: 101] Hawangener Feld genannt) keinen Zweifel. Die Entwicklung der oberen Hochterrasse fällt dabei nach SCHAEFER in das Altriß (R I seiner dreigeteilten Rißeiszeit); Graul nimmt dagegen eine frühe Phase der Rißmaximalvergletscherung (Mittelriß) für deren Entstehung an (Riß-Gliederung nach Graul 1952, 1953: Jungriß - Mittelriß - Altriß).

Die nachfolgenden Untersuchungen in der Gegend um Memmingen (SINN 1972: 107, 110-113; JERZ \& WAGNER 1978: 51, 54; HABBE \& RöGNER 1989b: 322) bestätigten das rißeiszeitliche Alter der Hochterrasse im Günztal.

LÖSCHER faßte hingegen (seit 1974) die GünztalHochterrasse als eine Ablagerung der Mindeleiszeit auf. Diese, durchaus nachzuvollziehende stratigraphische Einordnung stützte er auf folgende Untersuchungsergebnisse: Die Basis des Günztal-Hochterrassenschotters läßt sich am Vorfluter Donau hypsometrisch mit der des mindeleiszeitlichen KirchheimBurgauer Schotters im Mindeltal vergleichen (LÖSCHER \& LÉGER 1974: Abb. 3). Beide liegen etwa 15 m über dem Donautalboden. Die Basis des rißeiszeitlichen Sediments (Hochterrasse) im Mindeltal liegt dagegen um $10 \mathrm{~m}$ tiefer als die des sogenannten oberen Hochterrassenschotters im Günztal (LÖsCHER \& LÉGER 1974: 66). Wollte man die beiden Hochterrassen (sowohl die "tiefere" im Mindeltal als auch die „höhere“ im Günztal) in die Rißeiszeit stellen, hätte dies nach LÖsCHER (1976: 100-101) einen sehr kurvenreichen und damit eher unwahrscheinlichen Verlauf der Donau in dieser Zeit zur Folge gehabt. Zusätzlich wurde das mindeleiszeitliche Alter der Hochterrassenschotter im Günztal dadurch bekräftigt, daß dieser von derselben Fazies aufgebaut wird wie der Kirchheim-Burgauer Schotter im Mindeltal. Auch hinsichtlich der Intensität der Dolomitverwitterung besteht zwischen beiden weitgehende Übereinstimmung (LÖSCHER 1976: 98).

Außerdem versuchte LÖscher 1979 die oben genannte stratigraphische Einordnung des GünztalHochterrassenschotters mit pedologischen Argumenten aus dem Deckschichtenbereich zu erhärten. In der Arbeit Löscher et al. (1979) wurden Deckschichten bei Großkötz von Schies und Dabelstein und bei Autenried von LÉGER untersucht. Die LößDeckschichten sind dort jeweils in eine würm- und rißeiszeitliche Lößakkumulation zu untergliedern, so daß die darunterliegenden Schotter in die drittletzte Kaltzeit (Mindel) zu stellen waren (LÖsCHER et al. 1979: 180-188). Nach diesen Ergebnissen geht LöSCHER (1976: 101) zwangsläufig von einer vollständigen Ausräumung der rißeiszeitlichen Sedimente im Günztal durch nachfolgende Erosion aus.

1988 führten RÖGNER, LÖSCHER \& ZÖLLER erste Thermolumineszenz-Datierungen am Löß-Deckschichtenprofil Autenried durch. Die Datierung der Proben ergab für den liegenden Schotter ein mindestens mindeleiszeitliches Alter. In dieser Arbeit wurde erstmals eine Verbindung mit dem GrönenbachSchwaighausener Schotter im unteren Günztal vermutet (1988: 67).

\section{Ergebnisse}

Unsere vorliegenden Gelände- und Laborergebnisse zeigen, daß der in der Geologischen Übersichtskarte des Iller-Mindel-Gebiets 1:100000 (Jerz et al. 1975) als Hochterrasse ausgewiesene Bereich zwischen Günzburg und Tafertshofen differenzierter aufgebaut ist. Er gliedert sich in ein mindelzeitliches und ein rißzeitliches Niveau.

\subsection{Der mindeleiszeitliche Schotterkörper}

Der westlich der Günztal-Hochterrasse (Tafertshofen-Günzburger HT) liegende Mindelschotter besitzt von Unterwiesenbach bis Schneckenhofen ein Gefälle von etwa 3,3\% (Abb. 2: As.-Nr. 0151 und Nr. 0091, zu den Aufschlüssen siehe Tab. 1). Das Gefälle ist damit um $0,8 \% 0$ größer als das des parallel dazu verlaufenden Hochterrassenschotters (Abb. 2: As.-Nr. 0120 und 0090, zu den Aufschlüssen siehe Tab. 1). Die Schotterbasiswerte des Unterwiesenbach-Schneckenhofener Schotters können mit den- 


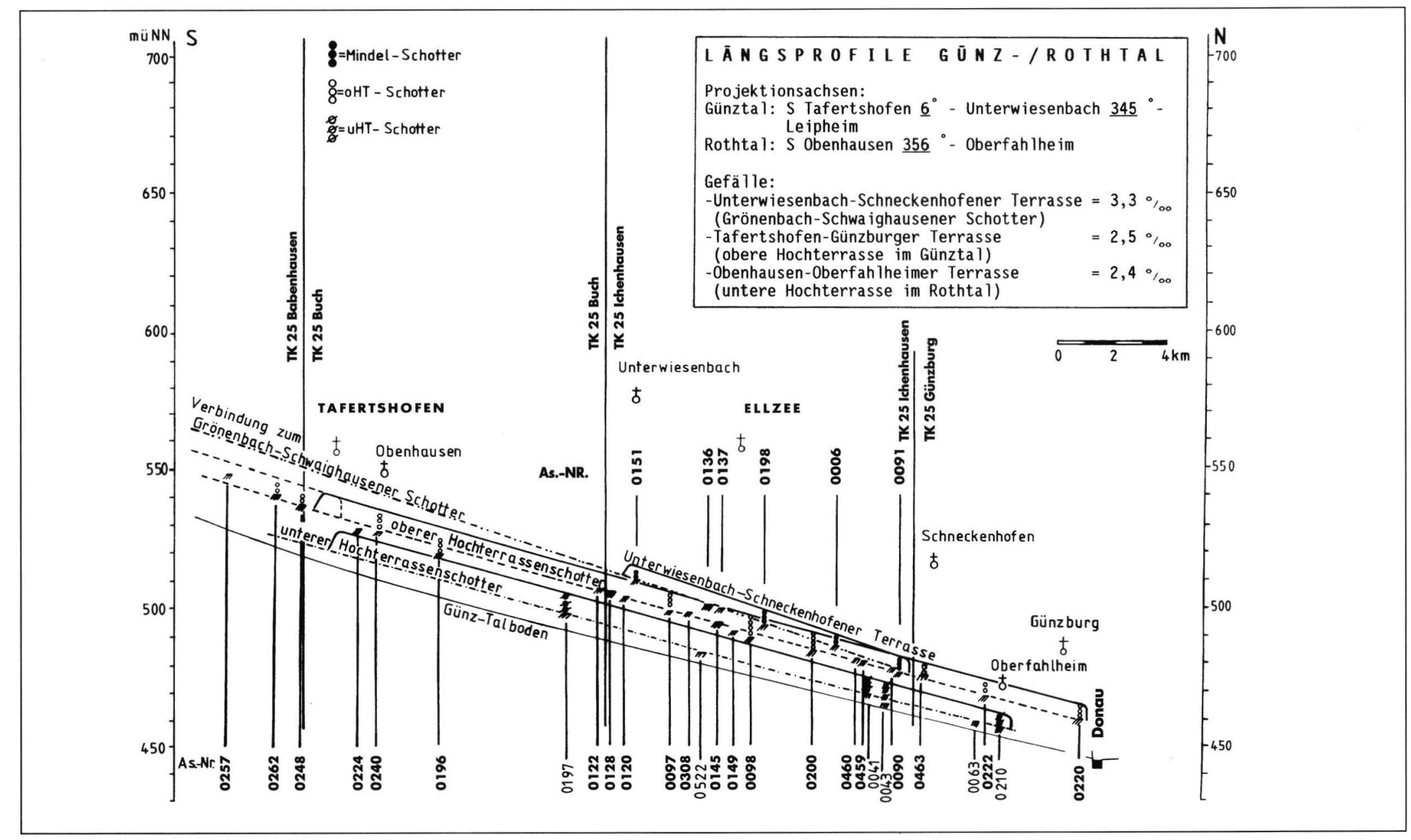

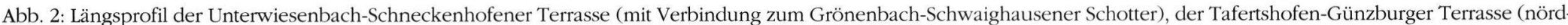

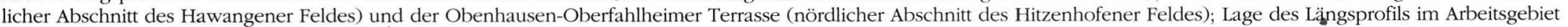
siehe Abb. 1, Aufschlußverzeichnis siehe Tab. 1

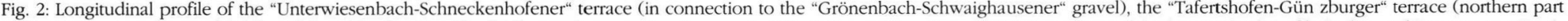

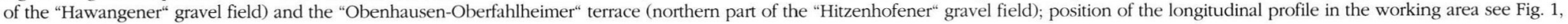
exposure datas see table 1 
Tab. 1: Aufschlußverzeichnis der im Text und in den Abbildungen angegebenen Lokalitäten. Angabe der Aufschlußnummer (As.-Nr.), des Aufschlußtyps (As.-Typ), des Rechts- und Hochwertes (RW/HW), der topographischen Karte im Maßstab 1:25000 (TK-Nr.), der Mächtigkeit der Deck-(I) und Kiesschicht (II), der Entkalkungstiefe im Schotter (III) sowie der Tertiär/Quartär(T/Q)-Grenze (IV). TK 25: 7526 = Ulm-Nordost, 7527 = Günzburg, $7626=$ Ulm-Südost, $7627=$ Ichenhausen, 7726 = Illertissen, $7727=$ Buch, $7827=$ Babenhausen. B = Bohrung, BGla = Bohrergebnisse des Geologischen Landesamtes München, $\mathrm{BFa}=$ Firmen-Bohrprotokolle, $\mathrm{Lgr}=$ Lehmgrube, $\mathrm{Kgr}=$ Kiesgrube, aSgr = zugewachsene Sandgrube, $522(\mathrm{LÖ})=\mathrm{T} / \mathrm{Q}-$ Grenze von Löscher (1976) übernommen bzw. zusammen mit LöschER 1991, 1992 im Gelände ermittelt, $(4,0)=$ keine Schotterlehmbildung, nur angewitterte Schotter; 5,1 (Mi) = Deckschichtenprofil im Hangenden der mindeleiszeitlichen Unterwiesenbach-Schneckenhofener Terrasse

Table 1: Catalog of exposure datas of the localities used in the text and figures. Number of the exposure (As.-Nr.), the type of exposure (As.-Typ), coordinates of the "Gauß-Krüger" map grid (RW/HW), specification (number) of the topographical map in the scale of $1: 25000$ (Tk-Nr.), thickness of the loess cover sediments (I) and of the gravel layer (II), the depth of decalcification in the gravel layers (III), tertiary/quaternary boundary in meters above sea level (IV). Topographical map number and location name: TK $25: 7526=$ Ulm-Nordost, $7527=$ Günzburg, $7626=$ Ulm-Südost, $7627=$ Ichenhausen, $7726=$ Illertissen, $7727=$ Buch, $7827=$ Babenhausen. $\mathrm{B}=$ drilling, BGla $=$ Results of the drillings of the Bavarian Geological Office, Munich; $\mathrm{BFa}=$ results of the drillings of several boring-companies, $\mathrm{Lgr}=$ mine of loam, $\mathrm{Kgr}=$ mine of gravels, aSgr $=$ shut down mine of sand, $522(\mathrm{Lo})=$ Tertiary/quaternary boundary by LöSCHER (1976) or measured together with Löscher in 1991, 1992, (4,0) = no loamy gravel, only weak weathered gravel, (Mi) loess cover sediments on the "Unterwiesenbach-Schneckenhofener" terrace (time of deposition of gravel = "Mindel"-glaciation)

Verzeichnis der Aufschlüsse/Bohrungen

\begin{tabular}{|c|c|c|c|c|c|c|c|}
\hline $\begin{array}{l}\text { As. - } \\
\mathrm{Nr} .\end{array}$ & $\begin{array}{l}\text { As } \\
\text { Typ }\end{array}$ & $\mathrm{RW} / \mathrm{HW}$ & $\begin{array}{l}\text { TK } 25 \\
\text { Nr. }\end{array}$ & $\begin{array}{c}\text { Deck- } \\
\text { schicht } \\
\text { in m } \\
\text { (I) }\end{array}$ & $\begin{array}{l}\text { Kies- } \\
\text { mächtig- } \\
\text { keit (m) } \\
\text { (II) }\end{array}$ & $\begin{array}{l}\text { Entkal- } \\
\text { kung } \\
\text { in m } \\
\text { (III) }\end{array}$ & $\begin{array}{l}\text { T/Q- } \\
\text { Gren- } \\
\text { ze m NN } \\
\text { (IV) }\end{array}$ \\
\hline 0006 & Lgr & $3593650 / 5359750$ & 7627 & $5,5(\mathrm{Mi})$ & 5,0 & & 484-8 (Lö) \\
\hline 0008 & B & $3589790 / 5367460$ & 7527 & 2,3 & & & \\
\hline 0014 & B & $3589480 / 5367280$ & 7527 & 3,05 & & & \\
\hline 0041 & $\operatorname{Kgr}$ & $3585140 / 5361700$ & 7626 & 1,0 & 7,0 & $(2,0)$ & 469,5 \\
\hline 0042 & Kgr & $3585340 / 5362360$ & 7626 & 0,8 & 13,2 & $(4,0)$ & 465,0 \\
\hline 0043 & Kgr & $3585340 / 5362340$ & 7626 & 1,5 & 12,5 & $(>2,0)$ & 465,0 \\
\hline 0045 & B & $3585690 / 5362550$ & 7626 & 1,4 & & & \\
\hline 0046 & B & $3585820 / 5362580$ & 7626 & 3,5 & & 0,5 & \\
\hline 0049 & $\mathrm{Kgr}$ & $3585160 / 5362400$ & 7626 & 1,6 & 12,4 & $(1,4)$ & 465,0 \\
\hline 0063 & B & $3584870 / 5365610$ & 7526 & 0,5 & & 0,5 & $<460$ (LÖ) \\
\hline 0065 & B & $3585760 / 5365820$ & 7526 & 0,75 & & 0,7 & \\
\hline 0067 & B & $3586650 / 5366160$ & 7527 & 2,0 & & $0 ; 45$ & \\
\hline 0069 & B & $3587170 / 5366420$ & 7527 & 1,1 & & & \\
\hline 0085 & B & $3594790 / 5362560$ & 7627 & 2,55 & & & \\
\hline 0086 & B & $3594590 / 5362450$ & 7627 & 2,7 & & & \\
\hline 0087 & B & $3594310 / 5362400$ & 7627 & 2,7 & & & \\
\hline 0088 & B & $3594100 / 5362340$ & 7627 & 2,9 & & 0,5 & \\
\hline 0090 & B & $3593670 / 5362360$ & 7627 & 0,6 & & & 478 (Lö) \\
\hline 0091 & B & $3593220 / 5362100$ & 7627 & $1,7(\mathrm{Mi})$ & 5,8 & & 476,5 ( LC் \\
\hline 0092 & B & $3593020 / 5362120$ & 7627 & $5,0(\mathrm{Mi})$ & & & \\
\hline 0093 & B & $3592800 / 5362170$ & 7627 & $5,2(\mathrm{Mi})$ & & & \\
\hline 0094 & B & $3592400 / 5362090$ & 7627 & $3,8(\mathrm{Mi})$ & & & \\
\hline 0096 & B & $3591780 / 5362160$ & 7627 & $4,0(\mathrm{Mi})$ & & & \\
\hline 0097 & Kgr & $3596300 / 5354600$ & 7627 & 1,9 & 5,1 & 1,0 & 498,0 \\
\hline 0098 & Kgr & $3597500 / 5357680$ & 7627 & 2,15 & 8,3 & 1,3 & 489,0 \\
\hline 0102 & B & $3595870 / 5358100$ & 7627 & 1,65 & & & \\
\hline 0103 & B & $3595630 / 5357910$ & 7627 & 1,8 & & & \\
\hline 0104 & B & $3585420 / 5357770$ & 7627 & 2,8 & & & \\
\hline 0106 & B & $3594890 / 5357535$ & 7627 & 3,55 & 5,0 & 0,25 & 490,0 \\
\hline 0108 & B & $3593090 / 5356940$ & 7627 & $5,7(\mathrm{Mi})$ & & & \\
\hline 0109 & B & $3593360 / 5356940$ & 7627 & $2,2(\mathrm{Mi})$ & & & \\
\hline 0112 & B & $3594400 / 5357370$ & 7627 & $5,6(\mathrm{Mi})$ & 5,4 & & 493,0 \\
\hline
\end{tabular}




\begin{tabular}{|c|c|c|c|c|c|c|c|}
\hline $\begin{array}{l}\text { As. - } \\
\mathrm{Nr} \text {. }\end{array}$ & $\begin{array}{l}\text { As } \\
\text { Typ }\end{array}$ & $\mathrm{RW} / \mathrm{HW}$ & $\begin{array}{l}\text { TK25 } \\
\mathrm{Nr} .\end{array}$ & $\begin{array}{l}\text { Deck- } \\
\text { schicht } \\
\text { in } \mathrm{m} \\
\text { (I) }\end{array}$ & $\begin{array}{l}\text { Kies- } \\
\text { mächtig- } \\
\text { keit (m) } \\
\text { (II) }\end{array}$ & $\begin{array}{l}\text { Entkal- } \\
\text { kung } \\
\text { in m } \\
\text { (III) }\end{array}$ & $\begin{array}{l}\text { T/Q- } \\
\text { Gren- } \\
\text { ze m NN } \\
\text { (IV) }\end{array}$ \\
\hline 0114 & B & $3589620 / 5367370$ & 7527 & 2,05 & & & \\
\hline 0115 & B & $3594250 / 5352650$ & 7627 & $5,1(\mathrm{Mi})$ & & 0,65 & \\
\hline 0116 & B & $3594630 / 5352645$ & 7627 & $4,7(\mathrm{Mi})$ & & & \\
\hline 0117 & B & $3595140 / 5352600$ & 7627 & $3,1(\mathrm{Mi})$ & & & \\
\hline 0118 & B & $3595620 / 5352560$ & 7627 & 2,7 & 2,8 & & 505,5 \\
\hline 0119 & B & $3595840 / 5352450$ & 7627 & 1,85 & 5,7 & & 505,5 \\
\hline 0120 & B & $3597900 / 5353200$ & 7627 & & & & 503,5 (Lö) \\
\hline 0122 & asgr & $3598080 / 5352280$ & 7627 & & & & 506,0 (Lö) \\
\hline 0124 & B & $3596370 / 5352500$ & 7627 & 2,6 & & & \\
\hline 0127 & B & $3596970 / 5352550$ & 7627 & 2,5 & & & \\
\hline 0128 & B & $3597900 / 5352540$ & 7627 & 2,5 & & & 505,0 \\
\hline 0134 & B & $3595630 / 5352550$ & 7627 & & & & 505,5 (Lö) \\
\hline 0136 & B & $3593840 / 5355240$ & 7627 & $0,7(\mathrm{Mi})$ & & & $50.1,0$ (LÖ) \\
\hline 0137 & B & $3592950 / 5355300$ & 7627 & $2,7(\mathrm{Mi})$ & & & 499,5 (Lö) \\
\hline 0145 & B & $3597220 / 5355400$ & 7627 & & & & 494,5 (Lö) \\
\hline 0149 & B & $3595810 / 5356470$ & 7627 & & & & 491,0 (Lö) \\
\hline 0151 & B & $3594920 / 5352680$ & 7627 & $1,35(\mathrm{Mi})$ & 2,65 & 1,4 & 509,5 (Lö) \\
\hline 0152 & B & $3593860 / 5352710$ & 7627 & $4,0(\mathrm{Mi})$ & & & \\
\hline 0196 & BGla & $3598360 / 5345680$ & 7727 & 2,0 & 6,3 & & 519,0 \\
\hline 0197 & BGla & $3586280 / 5350620$ & 7726 & 1,5 & 8,5 & 1,5 & 497,0 \\
\hline 0198 & BGla & $3594320 / 5357220$ & 7627 & $5,45(\mathrm{Mi})$ & 5,0 & & 493,0 \\
\hline 0199 & BGla & $3594990 / 5357400$ & 7627 & 1,9 & 5,0 & & 490,5 \\
\hline 0200 & BGla & $3597180 / 5359980$ & 7627 & 3,2 & 6,0 & & 484,0 \\
\hline 0206 & BGla & $3591960 / 5368160$ & 7527 & 4,5 & 4,5 & & 463,0 \\
\hline 0207 & BGla & $3591760 / 5368060$ & 7527 & 3,0 & 6,5 & & 463,5 \\
\hline 0210 & BGla & $3586300 / 5366620$ & 7526 & 1,0 & 4,4 & & 458,0 \\
\hline 0220 & $\mathrm{BFa}$ & $3595270 / 5369820$ & 7527 & 2,1 & 4,7 & & 460,0 \\
\hline 0222 & $\mathrm{BFa}$ & $3595770 / 5366220$ & 7527 & 1,4 & 5,0 & & 468,5 \\
\hline 0224 & B & $3594820 / 5343560$ & 7727 & & & & 528,0 (Lö) \\
\hline 0240 & $\operatorname{Kgr}$ & $3598020 / 5343480$ & 7727 & 1,8 & 6,5 & 1,2 & 527,0 \\
\hline 0248 & $\operatorname{Kgr}$ & $3594800 / 5341200$ & 7727 & 1,3 & 4,2 & 1,3 & 537,0 \\
\hline 0257 & Kgr & $3593560 / 5338520$ & 7827 & & & & 548,5 \\
\hline 0262 & $\mathrm{Kgr}$ & $3594460 / 5340000$ & 7827 & 0,7 & 4,0 & 1,0 & 540,5 \\
\hline 0267 & Kgr & $3596660 / 5362760$ & 7627 & 1,4 & 4,3 & & 477,0 \\
\hline 0308 & $\mathrm{~B}$ & $3596505 / 5355290$ & 7627 & & & & 497,5 \\
\hline 0347 & Lgr & $3593750 / 5359780$ & 7627 & $5,0(\mathrm{Mi})$ & & & \\
\hline 0440 & B & $3592130 / 5368370$ & 7527 & 5,1 & & & \\
\hline 0455 & B & $3592450 / 5368380$ & 7527 & 3,5 & & & \\
\hline 0459 & $\mathrm{BFa}$ & $3597380 / 5362030$ & 7627 & & & & 480,1 \\
\hline 0460 & $\mathrm{BFa}$ & $3597280 / 5361850$ & 7627 & & & & 481,0 \\
\hline 0461 & $\mathrm{BFa}$ & $3596440 / 5362860$ & 7627 & & & & 477,1 \\
\hline 0463 & $\mathrm{BFa}$ & $3596400 / 5363960$ & 7527 & 1,7 & 2,4 & & 475,6 \\
\hline 0467 & B & $3595490 / 5368340$ & 7527 & 3,0 & & & \\
\hline 0471 & B & $3595530 / 5369440$ & 7527 & 8,25 & & 0,9 & \\
\hline 0477 & B & $3586700 / 5355460$ & 7627 & 3,7 & & & \\
\hline 0522 & Kgr & $3586200 / 5355500$ & 7626 & & & & $<483$ (Lö) \\
\hline
\end{tabular}



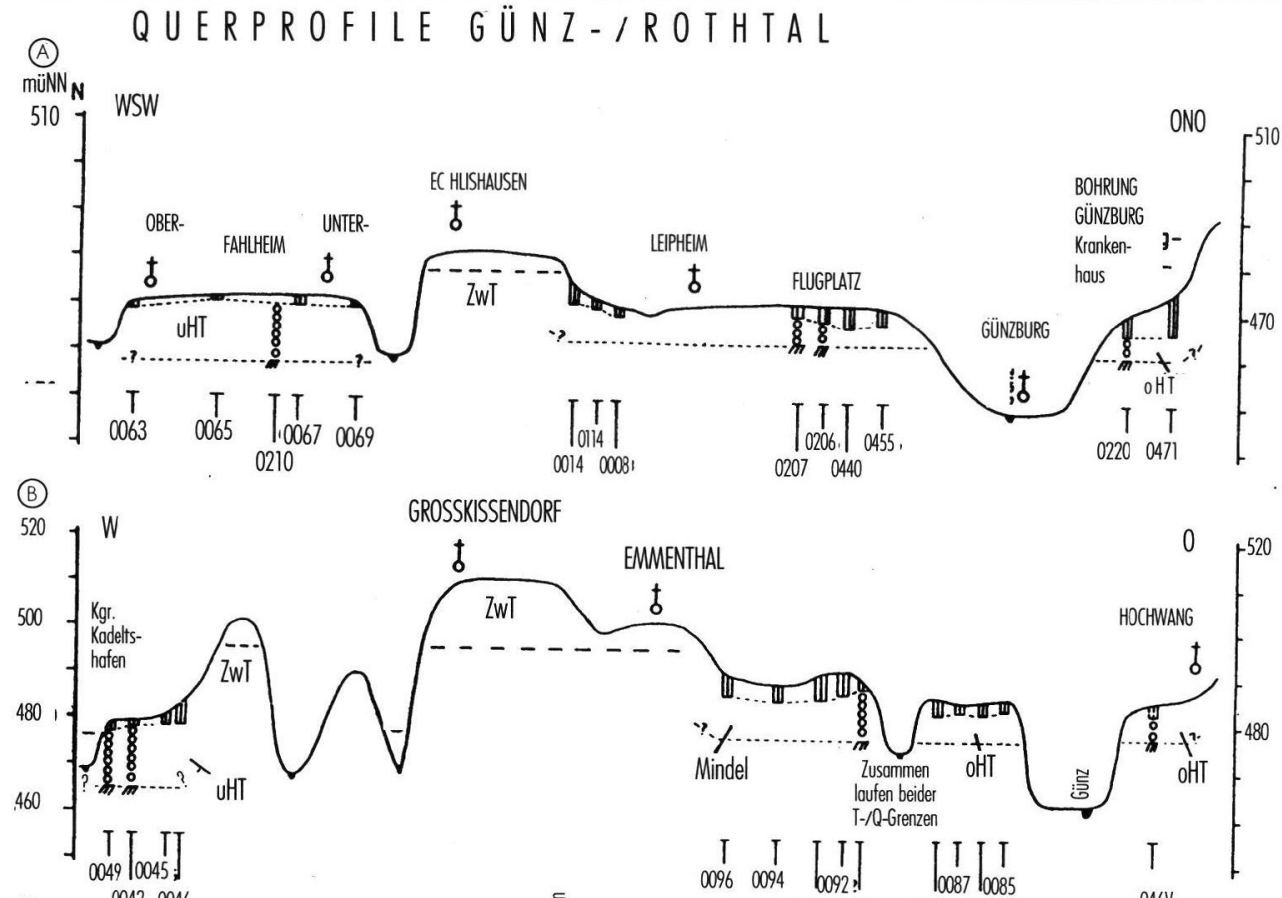

GROSSKISSENDORF

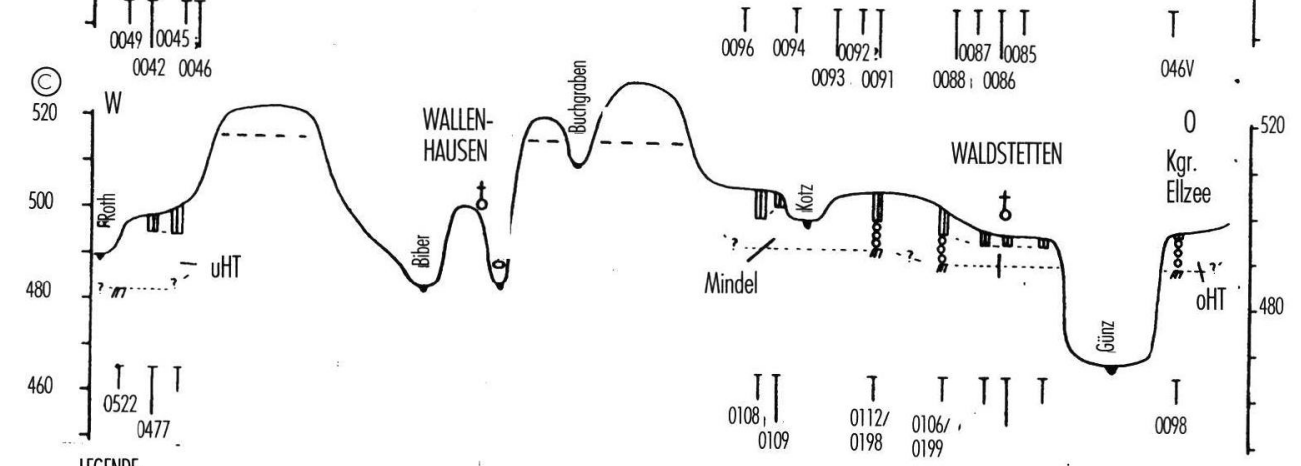

LEGENDE:

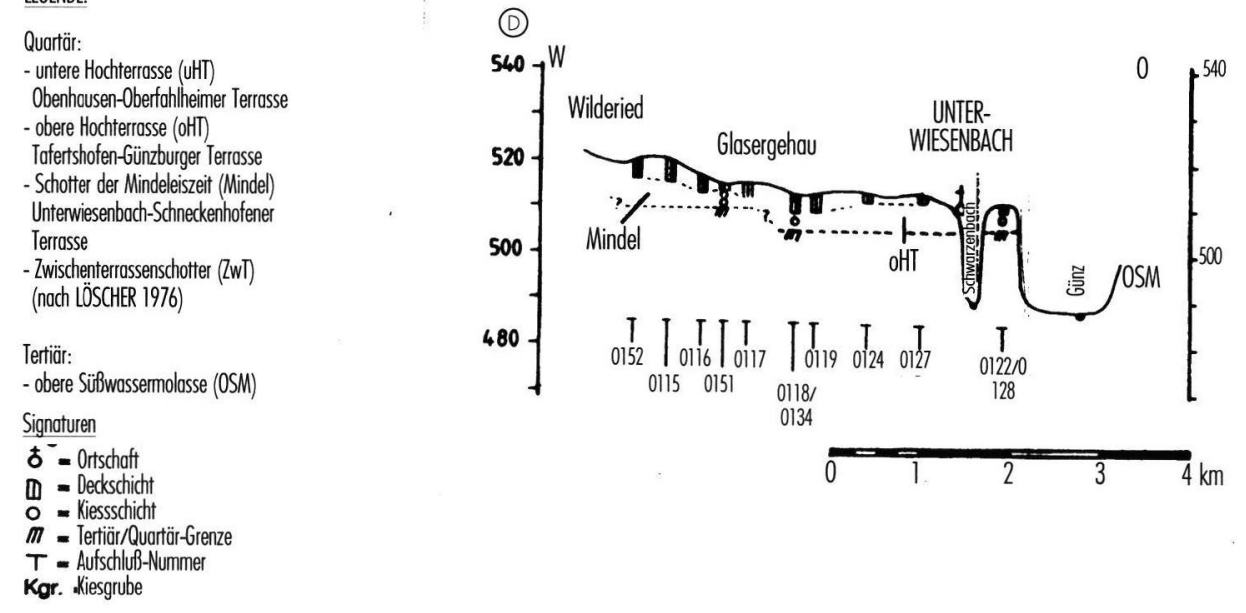

Abb. 3: Querprofile (A-D) im Günz- und Rothtal; Lage im Untersuchungsgebiet siehe Abb. 1, Aufschlußverzeichnis siehe Fig. 3: Cross profils (A to D) in the valley of the rivers Günz and Roth, position in the working area see Fig. 1, catalog of exposure datas see table 1 


\section{Deckschichtenprofil Autenried}

(Nr. 0006, 0347)

Tiefe in $\mathrm{m}$

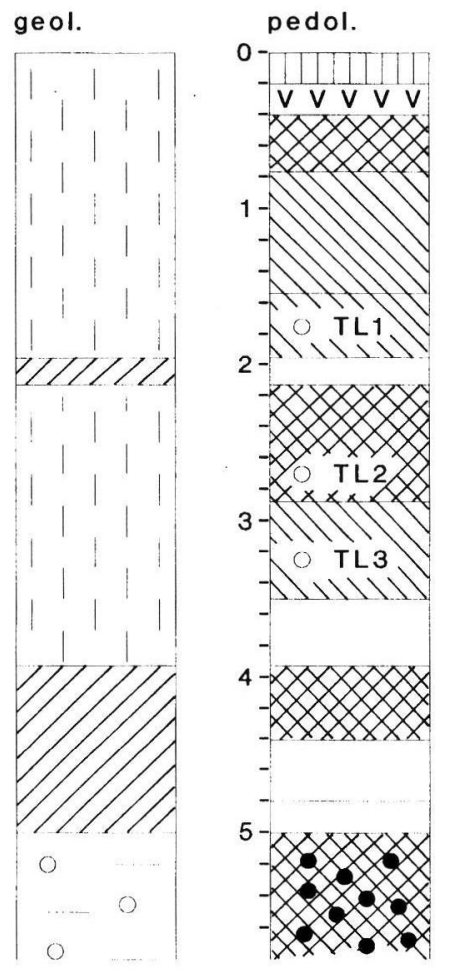

Ap, 10 YR $4 / 4$, humos

Al, lessiviert, aufgehellt

Bvt, 10 YR 5/8, Tonkutanen

SBvt, 10 YR 5/8, Tonkutanen

hydromorphe Spuren

SwBv, 10 YR 5/6, schwach tonig, Mn- und FeAusfällungen, TL-Alter: $52,5+-6,7 \mathrm{ka}$

ICv, gebändert, Mn-Knollen, FlieBerde

1.fSBvt, 10 YR 6/6, Tonkutanen, hydromorphe Spuren, oben plattiges Gefüge, TL-Alter: $131,0+-16,0 \mathrm{ka}$

SdBvt, 10 YR 5/6, Tonkutanen, Mn- und FeAusfällungen, TL-Alter: $142,0+-12,0 \mathrm{ka}$

BvCv, 10 YR 6/8, wenig Fe und.Mn, Tonkutanen

Bvt, 10 YR 5/8, wenig Gerölle, Feund $\mathrm{Mn}$-Konkretionen, Tonkutanen

ICv, skeletthaltig, Umlagerungshorizont

ICv, rötlich-braun, skeletthaltig

2.fSBvt, rot-braun, Schotterlehm in mindeleiszeitlichen Schottern

Thermolumineszenz(TL)-Alter aus:

ROGGNE, LOSCHER \& ZOLLER (1988)

\section{LEGENDE :}

\section{Geologie/Sedimentologie}

Aolische Sedimente:

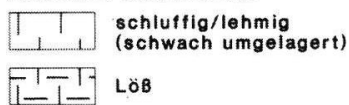

Fluviale Sedimente:

$0-0$

\section{glazifluvialer}

Hochterrassenkies
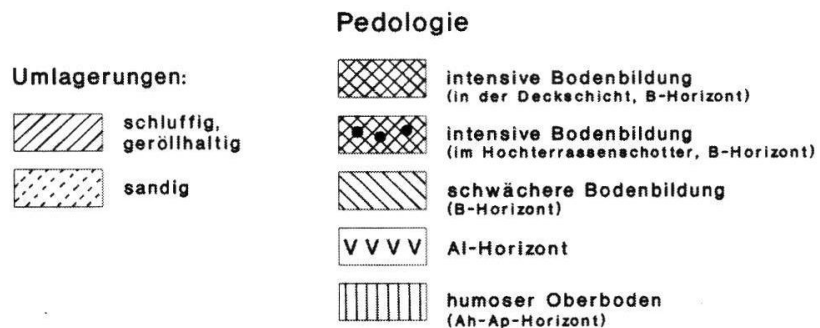

Abb. 4: Deckschichtenprofil in der Ziegeleigrube von Autenried mit Probennahmepunkte für die TL-Datierungen; Unterwiesenbach-Schneckenhofener Schotter aus der Mindeleiszeit (Aufnahme 1991, 1992)

Fig. 4: Profile of the cover sediments at the locality of Autenried with the points of sampling for the TL-datings; UnterwiesenbachSchneckenhofener gravel of the Mindel glaciation (years of survey: 1991, 1992)

jenigen des Grönenbach-Schwaighausener Schotters im Süden der Iller-Lech-Platte verbunden werden (= doppelt gestrichelt-gepunktete Linie in Abb. 2).
Bei Unterwiesenbach liegt die $\mathrm{T}-\mathrm{Q}$ Q-Grenze des mindeleiszeitlichen Schotters noch etwa $6 \mathrm{~m}$ über der des Hochterrassenschotters (Abb. 2: As.-Nr. 0151, 0120). 


\section{Profil Autenried}

Nr. 0006, 0347
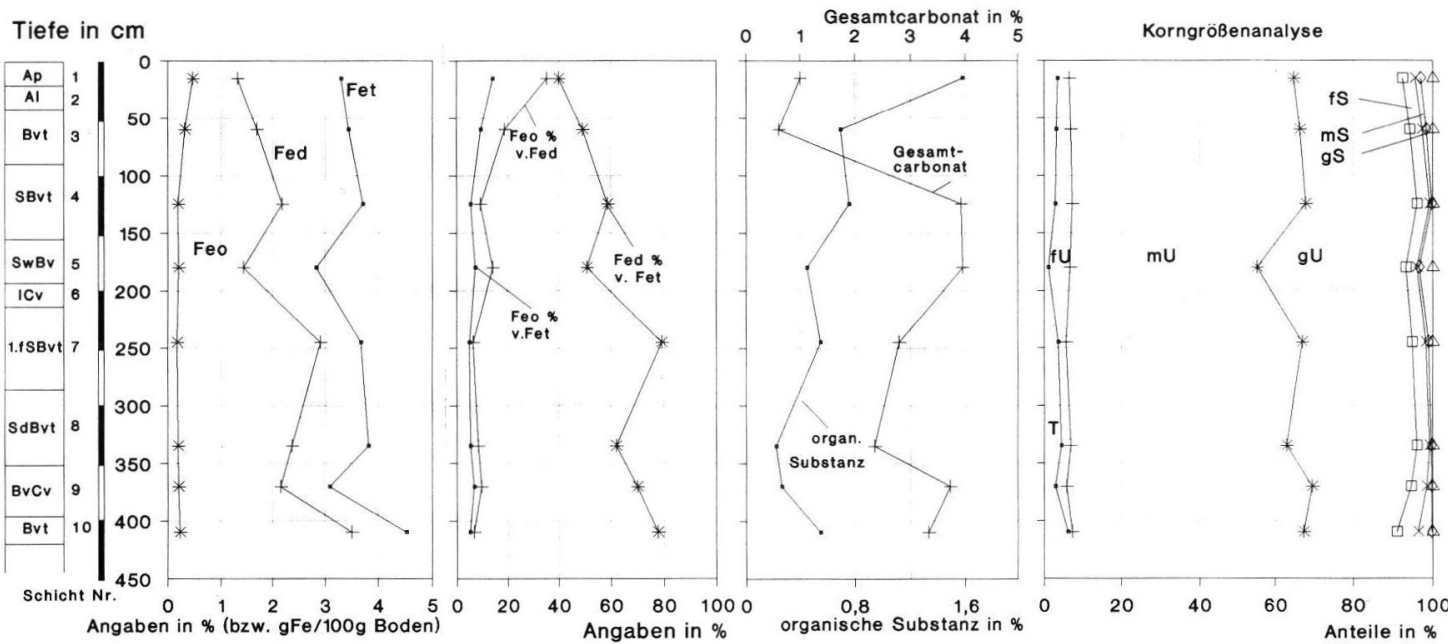

Abb. 5: Ergebnisse der Laboranalysen; Feo: oxalatlösliches Fisen, Fed: dithionitlösliches Eisen, Fet: Gesamteisen, Fed in $\%$ von Fet und Feo in \% von Fet: prozentuale Anteile verschiedener Eisenfraktionen am Gesamteisen, Feo in \% von Fed: prozentualer Anteil von oxalatlöslichem an dithionitlöslichem Eisen (= Aktivitätsgrad der Fe-Oxide nach SCHwERTMANN 1964), Korngrößenanalyse: $\mathrm{T}=$ Ton, $\mathrm{fU}=$ Feinschluff, $\mathrm{mU}=$ Mittelschluff, $\mathrm{gU}=$ Grobschluff, $\mathrm{fS}=$ Feinsand, $\mathrm{mS}=\mathrm{Mittel}-$ sand, $\mathrm{gS}=$ Grobsand

Fig. 5: Results of the laboratory investigations; Feo: $\mathrm{NH}_{4}$-Oxalat soluble iron-oxide (dyscrystalline iron-oxide), $\mathrm{Fed}_{\text {: }} \mathrm{Na}_{2} \mathrm{~S}_{2} \mathrm{O}_{4}$ soluble iron-oxide (whole pedological formed iron-oxide), Fet: total iron, Fed \% von Fet: percentage pedological iron-oxide of total iron, Feo $\%$ von Fet: percentage dyscrystalline iron-oxide of total iron; Feo \% von Fed: percentage dyscrystalline iron-oxide of pedological ironoxide, grain-size distribution: $\mathrm{T}=$ clay, $\mathrm{fU}=$ fine silt, $\mathrm{mU}=$ middle silt, $\mathrm{gU}=$ coarse silt, $\mathrm{fS}=$ fine sand, $\mathrm{mS}=$ middle sand, $\mathrm{gS}=$ coarse sand

Im Gebiet Schneckenhofen (ca. 10 km nördlich) konvergieren die 'Schotterbasiswerte scheinbar mit denen der Tafertshofen-Günzburger Hochterrasse (Abb. 3: Qpr. B, D). Dieses Verhalten ist Ergebnis eines unterschiedlichen Verlaufs des Vorfluters Donau; er lag im Mindelglazial deutlich südlicher als im Rißglazial.

Im Gegensatz zur etwa $2 \mathrm{~m}$ mächtigen Schotterverwitterung auf dem Rißschotter besitzt der Mindelschotter eine mindestens $3 \mathrm{~m}$ mächtige Verlehmungszone. Zudem sind darunter die Kalkgerölle stark angegriffen (Jerz \& DOPPLER 1990: 4). Nach Ergebnissen LÖsCHERS (1976: 98, 100) sind die Mindelschotter sogar bis zur tertiären Molasse entkalkt. Die deutlich unterschiedliche Verwitterung zeigt, daß es sich bei den beiden Schottern nicht um Teilfelder ein und derselben Vergletscherung handeln kann; beide Schotter repräsentieren Hauptfelder verschieden alter "Glazialer Serien“. Um diese Vermutung zu belegen, werden im folgenden die Deckschichtenprofile von Autenried (stärker verwitterter Schotter) und Günzburg (geringmächtigere Schotterverwitterung) vorgestellt.

\subsection{Deckschichtenprofil Autenried auf dem mindeleiszeitlichen Schotterkörper 3.2.1 Profilbeschreibung}

Der einzige größere Deckschichtenaufschluß existiert bei Autenried (Ziegelei). In dem dort aufge- schlossenen etwa $5 \mathrm{~m}$ mächtigen Profil (Abb. 4) treten neben der Bodenbildung des Holozäns (Ap-AlBvt: $0-0,8 \mathrm{~m}$ ) und der Mindel/Riß-Warmzeit (Schotterlehm $=2$. fSBvt: ab $5 \mathrm{~m}$ ) zwei weitere, intensiv rotbraun gefärbte Bereiche auf. Sie werden ebenfalls als Böden angesprochen (1. fSBvt, Bvt: 2,1-2,9 m und 3,9-4,4 m). Ihre intensive Entwicklung wird durch Tonbeläge, eine intensive Eisen- und Manganfreisetzung sowie das fein- und grobpolyedrische Gefüge nachgewiesen.

Unter Berücksichtigung der morphostratigraphischen Ergebnisse (Kap. 3.1), der relativ geringmächtigen Lößakkumulation in diesem Gebiet sowie der Ausprägung des 1. fossilen Bodens, wird das Decksediment in ein Würm- und Rißlößpaket gegliedert (so auch LÉGER in LÖSCHER et al. 1979: 183; RöGNER et al. 1988: 67-68). In dem nur $2 \mathrm{~m}$ mächtigen Würmlößsediment hat sich im Holozän eine Parabraunerde (Ap-Al-Bvt) gebildet. Im Liegenden der holozänen Bodenbildung sind zwei weitere schwächer ausgebildete B-Horizonte erhalten geblieben (SBvt, SwBv: 0,8-1,9 m). Eine Einstufung dieser B-Horizonte in die von SCHÖNHals et al. (1964), Semmel (1968) oder BrunNacker (1957) vorgeschlagenen Abschnitte des Würm war nicht möglich. Wahrscheinlich haben wegen der geringen Sedimentmächtigkeit (kaltzeitliche Denudation in Verbindung mit geringer Sedimentation) ver- 
schiedene Bodenbildungen auf dasselbe Sediment eingewirkt, so daß verwertbare Leithorizonte ( $\mathrm{z}$. B. Humuszonen) nicht erhalten geblieben sind.

Der 1. fossile Boden (1. fSBvt-Horizont: 2,1 - 2,9 m) ist auch makroskopisch deutlich im Profil zu erkennen. Außerdem zeigen Tonbeläge und das polyedrische Gefüge eine parabraunerdeartige Bodenentwicklung. Dieser Boden wurde im Riß/Würm-Interglazial unter warmzeitlichen Bedingungen gebildet. Die Pseudovergleyung entwickelte sich entweder zum Beginn des Würmglazials oder bereits in einer feuchten Phase der ausklingenden Riß/WürmWarmzeit (so ROHDENBURG \& Meyer 1979: 76 an Lößprofilen in Niedersachsen). Durch die Pseudovergleyung ist ein Vergleich mit dem Homburger Boden und dessen Äquivalent in Nordhessen (SchöNHALs et al. 1964: 200-201) möglich. Der Umlagerungshorizont im Hangenden des 1. fossilen Bodens (ICv: 1,9-2,1 m) geht auf Solifluktionsprozesse während der ersten kaltzeitlichen Abschnitte des Würm zurück. Unterhalb des SdBvt und BvCv-Horizonts ist im Bvt-Horizont (3,9-4,4 m) eine weitere

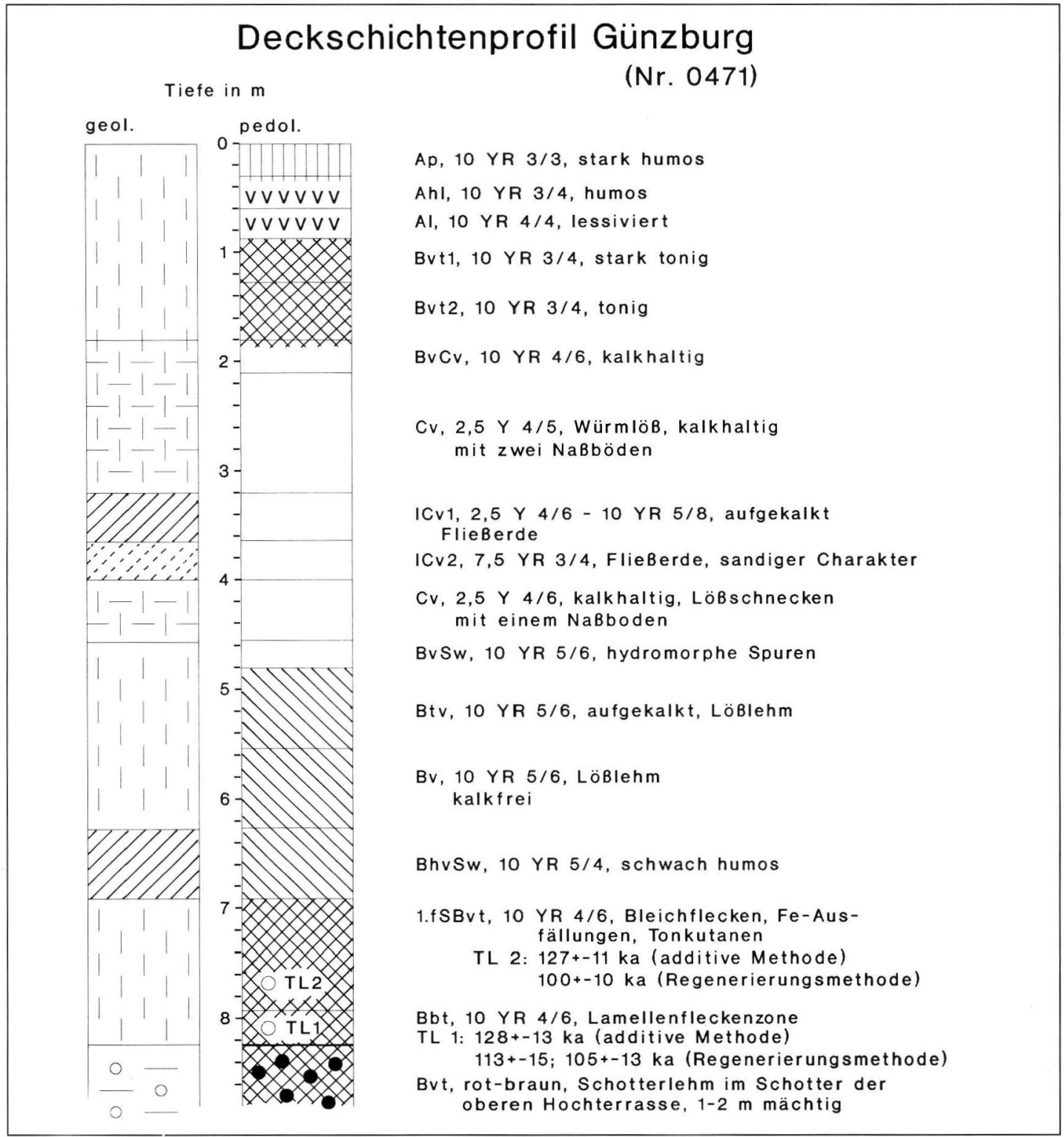

Abb. 6: Deckschichtenprofil nach den Ergebnissen der Bohrung am Krankenhaus Günzburg mit Probennahmepunkten für die TL-Datierungen, Legende siehe Abb. 4, Tafertshofen-Günzburger Hochterrasse (Aufnahme 1992)

Fig. 6: Profile of the cover sediments at the locality of Günzburg with the points of sampling for the TL-datings; legend see fig. 4, Tafertshofen-Günzburger high terrace (year of survey 1992) 
stärkere (zweite) Bodenbildung innerhalb des Rißlösses zu vermuten. Sie kommt in ausgeprägten Tonkutanen, Tonansammlungen in Hohlräumen und durch eine intensive Eisen- und Manganfreisetzung zum Ausdruck. Aufgrund der vereinzelt auftretenden Gerölle könnte hier eine Bodenbildung vor oder nach einer kurzen Umlagerung auf dieses Sediment eingewirkt haben. Stratigraphisch ließe sich diese Bodenbildung mit dem Innerriß-Schotterlehm in Baltringen-Ost (MiARA et al. 1996) vergleichen. Zu zweigeteilten Rißlößgliederungen kamen auch LÉGER (1987: 612) am Profil Roßhaupten und BRUNNACKER (1958: 139) am Profil Kitzingen. Eine detailliertere Untergliederung des Rißlösses wie von BıBus (1974: 179) im Rhein-Main-Gebiet vorgenommen, ist hingegen in Autenried nicht möglich.

\subsubsection{Laborergebnisse}

Die im Labor gewonnenen Ergebnisse (Abb. 5) unterstützen die Geländeansprache der Deckschichten. Die holozäne Bodenbildung (Ap-Al-Bvt: Schichten 1-3) ist in dem noch schwach carbonathaltigen Würmlöß (ca. $4 \%$ Gesamtcarbonat) ausgebildet. Sie kommt in den Anteilen an organischer Substanz und im hohen Aktivitätsgrad der Fe-Oxide (Aktivitätsgrad nach SCHwertmann 1964) zum Ausdruck. Die Bodenbildungen des Würm (SBvt, SwBv: Schichten $4,5)$ lassen sich in den prozentualen Anteilen an organischer Substanz erkennen. Der 1. fossile Boden des Riß/Würm-Interglazials (1. fSBvt: Schicht 7) sowie eine weitere Bodenbildung (Bvt: Schicht 10), die einer frühen Phase des Rißglazials zuzuordnen ist, sind am Gehalt des kristallinen Eisens (Fed) sowie an dessen prozentualem Anteil am Gesamteisen (Fed in \% von Fet) auszumachen. Zusätzlich weisen die Gehalte der organischen Substanz sowie die der Tonfraktion Bereiche ehemaliger Bodenentwicklungen aus.

Auch die von SCHIES und DABELSTEIN (in LÖSCHER et al. 1979: 184) mitgeteilten Labordaten deuten auf eine weitere Bodenbildung in der Frühphase des Rißglazials unterhalb des 1. fossilen Bodens hin (deren Horizont IIC2, Probe 17: erhöhter Tongehalt und erhöhte organische Substanz, geringer Carbonatgehalt). In der pedostratigraphischen Gliederung wird diese Entwicklung von ihnen jedoch nicht als Bodenbildung angesprochen.

\subsection{Der rißeiszeitliche Schotterkörper}

Für den mit dem Hawangener Feld zu verbindenden Hochterrassenschotter läßt sich von Tafertshofen bis Günzburg ein Gefälle von 2,5\% errechnen (Abb. 2). Die durchschnittliche Schottermächtigkeit liegt zwischen 4 und $6 \mathrm{~m}$. Eine Ausnahme bildet das Gebiet um Ellzee. Dort wurde eine Schottermächtigkeit von mehr als 8 m (As.-Nr. 0098, siehe Tab. 1) festgestellt. Diese Veränderung in der Mächtigkeit wie auch die Erhaltung des mindeleiszeitlichen Schotters kann strömungsdynamisch erklärt werden. Durch das seitliche Ausbiegen des Schmelzwasserstroms nach Osten entstand eine Prallhangsituation bei Ellzee. Tiefen- und Seitenerosion räumten dabei sogar den älteren Deckschotter am Hungerberg aus. In der Gleithangsituation, d. h. im Westen, konnte deshalb der Unterwiesenbach-Schneckenhofener Schotter aus der Mindeleiszeit nicht vollständig erodiert werden.

\subsection{Das Deckschichtenprofil Günzburg auf der rißeiszeitlichen Hochterrasse}

\subsubsection{Profilbeschreibung}

Die obere Hochterrasse ist von Tafertshofen bis nördlich Großkötz von $3 \mathrm{~m}$ mächtigen, wenig gegliederten äolischen Sedimenten bedeckt. Die Gründe für diese geringe Mächtigkeit sind (wie bei Autenried) in den ungünstigen Entstehungs- und Erhaltungsbedingungen zu suchen (siehe Kap. 3.2.1).

Im nördlichsten Teil der oberen Hochterrasse ändern sich jedoch diese Bedingungen aufgrund der Nähe zum Donautal, das als Auswehungsgebiet diente; es entstanden mächtige, durch Bodenbildungen gegliederte Löß-Deckschichten.

Da zur Zeit keine Aufschlüsse existieren, wurde das Deckschichtenprofil Günzburg (Abb. 6) durch Sondierungen mit dem Peilstangenbohrgerät vorerkundet und das Probengut mit einem Flügelbohrer ( $5 \mathrm{~cm}$ Durchmesser) gewonnen.

Die intensivsten Bodenbildungen des Profils Günzburg sind die rezente Parabraunerde (Ap-Ahl-Al-Bvt 1,2: $0-1,8 \mathrm{~m})$, der Schotterverwitterungslehm im Hochterrassenschotter (Bvt: ab 8,3 m) sowie der 1. fossile Boden (1. fSBvt-Bbt: 6,9-8,3 m) im Hangenden des Schotterlehms. Daneben treten schwächere Bodenbildungen (Btv, Bv, BhvSw: 4,8-6,9 m) sowie drei Naßböden im Würmlöß (in den beiden Cv-Horizonten: $2,1-3,2 \mathrm{~m}$ und 4-4,5 $\mathrm{m}$ ) auf. Die stratigraphische Gliederung der Deckschichten erfolgt mit Hilfe des 1. fossilen Bodens. Er befindet sich im unteren Abschnitt der Löß-Deckschicht als 1. fSBvt und wird von einer Lamellenfleckenzone (i. S. LIEBEROTHS 1959: 146-154) unterlagert (Bbt).

Der 1. fSBvt-Horizont mit einem Subpolyedergefüge weist die für Parabraunerden typischen Tonbeläge an den Aggregatoberflächen auf. Für die Entwicklung dieses fossilen Bt-Horizontes mit ausgeprägter Tonverlagerung aus den (bereits wieder erodierten) Ah- und Al-Horizonten kann nur ein Klima verantwortlich gemacht werden, das dem holozänen entspricht. Da bisher im Inner-Würm keine derartig ausgeprägten Warmzeiten nachgewiesen sind, ist 
der 1. fSBvt im Eem (Riß/Würm-Interglazial) gebildet worden. Er ist damit ein Äquivalent zum Homburger Boden i. S. SchÖNHALs' et al. (1964: 200) sowie zum Abschnitt R/Wb i. S. BRUnNaCKERs (1957: 90).

Eine ähnliche Profildifferenzierung wie bei Günzburg konnten auch BENTE \& SCHWEIZER (1988: 8-11, 15-16) an den Löß-Profilen südlich Heidelberg nachweisen. In ihren Profilen geht ebenso wie im Profil Günzburg der Bt-Horizont der Parabraunerde nach unten in eine Bänderparabraunerde (Bbt) über.

Die Pseudovergleyung der fossilen Parabraunerde im Profil Günzburg hat sich in einer klimatisch feuchteren Phase zum Ende des Riß/Würm-Interglazials oder zum Beginn des Würmglazials eingestellt. An süddeutschen Pollenprofilen konnte bereits BLuDAU (1995) auf klimatisch feuchtere Verhältnisse zum ausgehenden Eem-Interglazial hinweisen.

Im Liegenden des 1 . fossilen Bodens folgt unterhalb der Lamellenfleckenzone eine weitere intensive Bodenbildung im Hochterrassenschotter, der Schotterverwitterungslehm (Bvt). Hier sind die ehemals carbonathaltigen glazifluvialen Schotter in situ verwittert. Die mit der Bodenbildung einhergehende Verlehmung und Verbraunung äußert sich in einer rotbraunen und tonigen Matrix. Für eine derartige Schotterverwitterung sind ebenfalls warm- zeitliche Bodenbildungsbedingungen notwendig. Der 1. fossile Boden, durch seine stratigraphische Position als Eem-Boden ausgewiesen, hat sich in einem lößartigen Sediment gebildet, das nach den später vorzustellenden TL-Daten (siehe Kap. 3.5) in einer jüngeren Phase der Rißeiszeit abgelagert wurde. Da der untere Teil des 1. fossilen Bodens noch einen geringen Kalkgehalt aufweist (Bbt-Horizont: 2,5\% Gesamtcarbonat, siehe Abb. 7 und Kap. 3.4.2), muß der unterhalb des Bbt-Horizontes aufgefundene Schotterlehm als Bildung einer der Eem vorangegangenen Warmzeit mit ebenfalls intensiver Bodenbildung angesprochen werden. Der Hochterrassenschotter, in welchem sich der Schotterlehm ausbildete, ist wie der untere Teil der Löß-Deckschicht ebenfalls eine Ablagerung der Rißeiszeit (vgl. Kap. 2).

Diese Sachverhalte lassen nur den Schluß zu, daß der Hochterrassenschotter in einer früheren Phase der Rißeiszeit abgelagert wurde und in einer InnerRißphase mit warmzeitlichem Charakter zu_Schotterlehm verwitterte, während in einer darauffolgenden späteren Rißphase der liegende Teil des Deckschichtenpaketes zur Akkumulation kam. Damit ist eine Zweiteilung des Rißglazials, unterbrochen von einem Interglazial, vorzuschlagen (so auch BRunN-

\section{Profil Günzburg Nr. 0471}

Tiefe in $\mathrm{cm}$

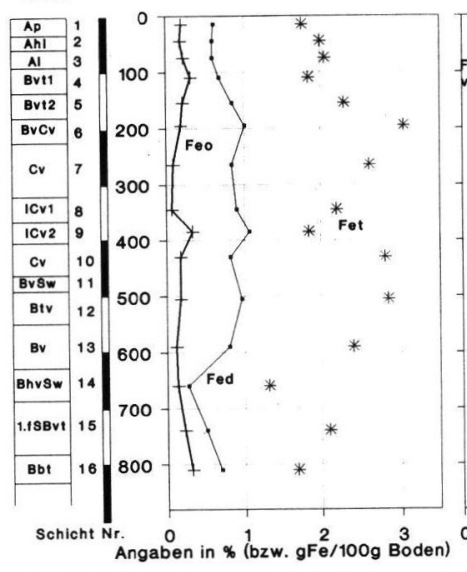

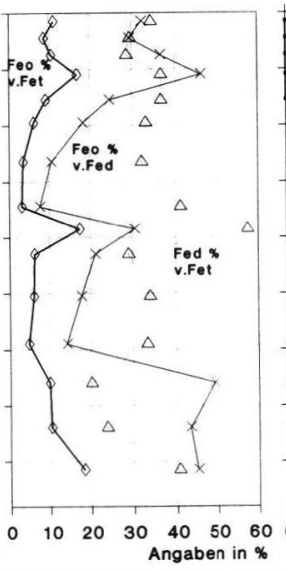

organische Substanz in \%

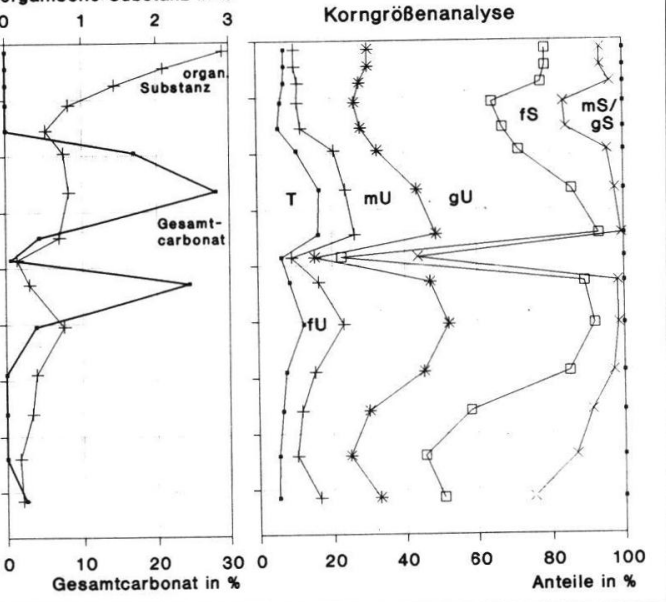

Abb. 7: Ergebnisse der Laboranalysen; Feo: oxalatlösliches Eisen, Fed: dithionitlösliches Eisen, Fet: Gesamteisen, Fed in $\%$ von Fet und Feo in \% von Fet: prozentuale Anteile verschiedener Eisenfraktionen am Gesamteisen, Feo in \% von Fed: prozentualer Anteil von oxalatlöslichem an dithionitlöslichem Eisen (= Aktivitätsgrad der Fe-Oxide nach SCHWERTMANN 1964), Korngrößenanalyse: $\mathrm{T}=\mathrm{Ton}, \mathrm{fU}=$ Feinschluff, $\mathrm{mU}=$ Mittelschluff, $\mathrm{gU}=$ Grobschluff, $\mathrm{fS}=$ Feinsand, $\mathrm{mS}=\mathrm{Mittel}-$ sand, gS = Grobsand

Fig. 7: Results of the laboratory investigations; Feo: $\mathrm{NH}_{4}$-Oxalat soluble iron-oxide (dyscrystalline iron-oxide), Fed: $\mathrm{Na}_{2} \mathrm{~S}_{2} \mathrm{O}_{4}$ soluble iron-oxide (whole pedological formed iron-oxide), Fet: total iron, Fed \% von Fet: percentage pedological iron-oxide of total iron, Feo $\%$ von Fet: percentage dyscrystalline iron-oxide of total iron; Feo \% von Fed: percentage dyscrystalline iron-oxide of pedological ironoxide, grain-size distribution: $\mathrm{T}=$ clay, $\mathrm{fU}=$ fine silt, $\mathrm{mU}=$ middle silt, $\mathrm{gU}=$ coarse silt, $\mathrm{fS}=$ fine sand, $\mathrm{mS}=$ middle sand, $\mathrm{gS}=$ coarse sand 
ACKER (1957: 85-86) an Lößprofilen nördlich Regensburg). Möglicherweise hat die Schotterlehmbildung in Günzburg zeitgleich mit der Bodenbildung im Bvt-Horizont (3,9-4,4 m, Abb. 4) im Deckschichtenprofil Autenried stattgefunden (vgl. dazu Kap. 4).

Im Hangenden der fossilen Pseudogley-Parabraunerde (1. fSBvt) folgen die schwächer ausgebildeten Böden wärmerer Phasen des Würm. Nach der Farbund Gefügeausprägung sowie der stratigraphischen Position wird der untere grau-braune Horizont (BhvSw: 6,3-6,9 m) dem Altwürm und die darüber folgenden (Btv, Bv: 4,8-6,3 m) dem Mittelwürm zugeordnet.

Der von 4,8-5,7 $\mathrm{m}$ reichende Btv-Horizont kann als Pendant zum „Lohner Boden“ (SCHÖNHALS et al. 1964: 203) und zum WI/WII-Abschnitt (BRUNNACKER 1956: 4) interpretiert werden.

Der carbonathaltige Jungwürmlöß (Cv: 2,1-3,2 und 4$4,5 \mathrm{~m})$ zeichnet sich durch die hochkaltzeitliche Schneckenfauna succinea oblonga aus. Aufgrund geringer Wärmeschwankungen im Verlauf des Jungwürm bildeten sich drei Tundrennaßböden aus (möglicherweise äquivalente Bildungen zu den Erbenheimer Naßböden i. S. SCHÖNHALS' et al. 1964: 203).

\subsubsection{Laborergebnisse}

Die holozäne Bodenbildung (eine humose Parabraunerde) im stark carbonathaltigen Jungwürmlöß läßt sich am erhöhten Aktivitätsgrad der Fe-Oxide (Feo \% v. Fed) sowie am erhöhten Umsatz von Sekundäreisen am Gesamteisen (Fed \% von Fet) erkennen (siehe Abb. 7).

Die Mittelwürmbodenbildungen (Btv, Bv: Schichten $12,13)$ kommen in den leicht erhöhten Fed-Werten sowie im Umsatz von pedogen entstandenem Eisen am Gesamteisen (Fed in \% von Fet) zum Ausdruck. Die mit der Verbraunung einhergehende Verlehmung (Btv: Schicht 12) spiegelt sich im erhöhten Tongehalt (Korngrößenanalyse) wider.

In den Mittelwürmböden konnten verhältnismäßig hohe Mengen an organischer Substanz ermittelt werden. Vergleichbar hohe Werte stellten auch BENTE \& LÖSCHER (1987: 13) in den oberen Mittelwürmböden (Lohner Boden) in den Profilen Nußloch und Rotenberg (südlich Heidelberg) fest.

Der hohe Umsatz von Sekundäreisen am Gesamteisen (Fed in \% von Fet) weist insbesondere im Bbt-Horizont (7,9-8,3 m: Schicht 16) unterhalb des 1. fossilen Bodens auf eine Bodenbildung hin. Es ist anzunehmen, daß in diesen Horizonten die Verteilung der FeGehalte nicht nur pedogen, sondern auch sedimentogen geprägt ist (vgl. dazu HäDricH 1970: 129-131).

Der noch sehr geringe Carbonatgehalt im Bbt-Horizont verdeutlicht, daß der angewehte, ehemals carbonathaltige Rißlöß während des Eem-Interglazials nicht vollständig entkalkt ist. Daraus kann geschlos- sen werden, daß für die Schotterlehmbildung (Bvt: ab $8,3 \mathrm{~m}, \mathrm{Abb} .6$ ) und die fossile Pseudogley-Parabraunerde (1. fSBvt-Bbt) nicht ein und derselbe Bodenbildungszeitraum in Frage kommen kann.

Ein Durchgriff der Bodenbildung durch die Deckschicht in den Hochterrassenschotter im Verlauf der Eem-Warmzeit muß nach diesen Ergebnissen abgelehnt werden.

\subsection{Thermolumineszenz (TL)-Datierungen an den Deckschichtenprofilen Günzburg und Autenried}

Zusätzlich zu den Gelände- und Laboruntersuchungen wurden an den Löß-Deckschichten in Autenried (vgl. RöGNER et al. 1988) und Günzburg TL-Datierungen durch L. ZÖLLER 1988 und 1993 durchgeführt.

Die Daten bestätigen die durch Gelände- und Laborbefunde vorgenommenen pedostratigraphischen Gliederungen der beiden Profile. Das rißeiszeitliche Alter des Sediments, in dem sich der 1. fossile Boden gebildet hat, wird durch die Ergebnisse der TL-Probe 2 für Autenried (131,0 $16,0 \mathrm{ka})$ und die Proben 1 und 2 für Günzburg $(127 \pm 11,128 \pm 13 \mathrm{ka}$, additive Methode) bestätigt (siehe Abb. 4, 6). Die Ergebnisse weisen durchschnittliche TL-Alter von $130 \mathrm{ka}$ aus. Demzufolge handelt es sich nicht um Würm-, sondern um Rißlöß. Die in diesen Substraten festgestellten kräftigen Bodenbildungen stammen folglich aus dem Riß/Würm-Interglazial.

Nach den gemessenen TL-Sedimentationsaltern (ca. $130 \mathrm{ka})$ lassen sich die oben genannten Böden mit den Riß/Würm-Interglazialböden in den Profilen Offingen (RÖGNER et al. 1988: 67-68 = 121 $\pm 12 \mathrm{ka}$ ) und Baltringen-Ost (MiARA et al. $1996=106 \pm 20,150 \pm 22$ ka) parallelisieren. Zudem entsprechen die 1. fossilen Böden in Günzburg und Autenried der Stufe $5 \mathrm{e}$ $(128-116 \mathrm{ka} \mathrm{BP}=\mathrm{Eem})$ der marinen Sauerstoffisotopen-Chronologie (vgl. Turon 1984: 674-675; VANVliet Lanoe 1989: 110; Pécsi 1991: 146).

Unterhalb des 1. fossilen Bodens folgen im Profil Autenried die Rißlößschichten. Für sie wurde ein TLAlter von $142 \pm 12 \mathrm{ka}$ ermittelt (TL 3, Abb. 4).

Im Hangenden des Riß/Würm-Bodens läßt das Mittelwürmalter in der Deckschicht Autenried (53,5 $\pm 6,7$ ka, TL 1, SwBv-Horizont, Abb. 4) einen Vergleich mit dem im Profil Rotenberg (53,3 $\pm 51 \mathrm{ka}$; südlich Heidelberg) zu. Für den SwBv-Horizont in Autenried könnte nach den Ergebnissen ZÖLLERS (in ZÖLLER et al. 1988: 55; 1989: 107) eine dem Gräselberger Boden (i. S. SchÖNHALS' et al. 1964: 201) parallele Entwicklung angenommen werden.

\section{Zusammenfassung und Diskussion}

Die vorgelegten morpho- und pedostratigraphischen Ergebnisse sowie die Ergebnisse der TL-Da- 


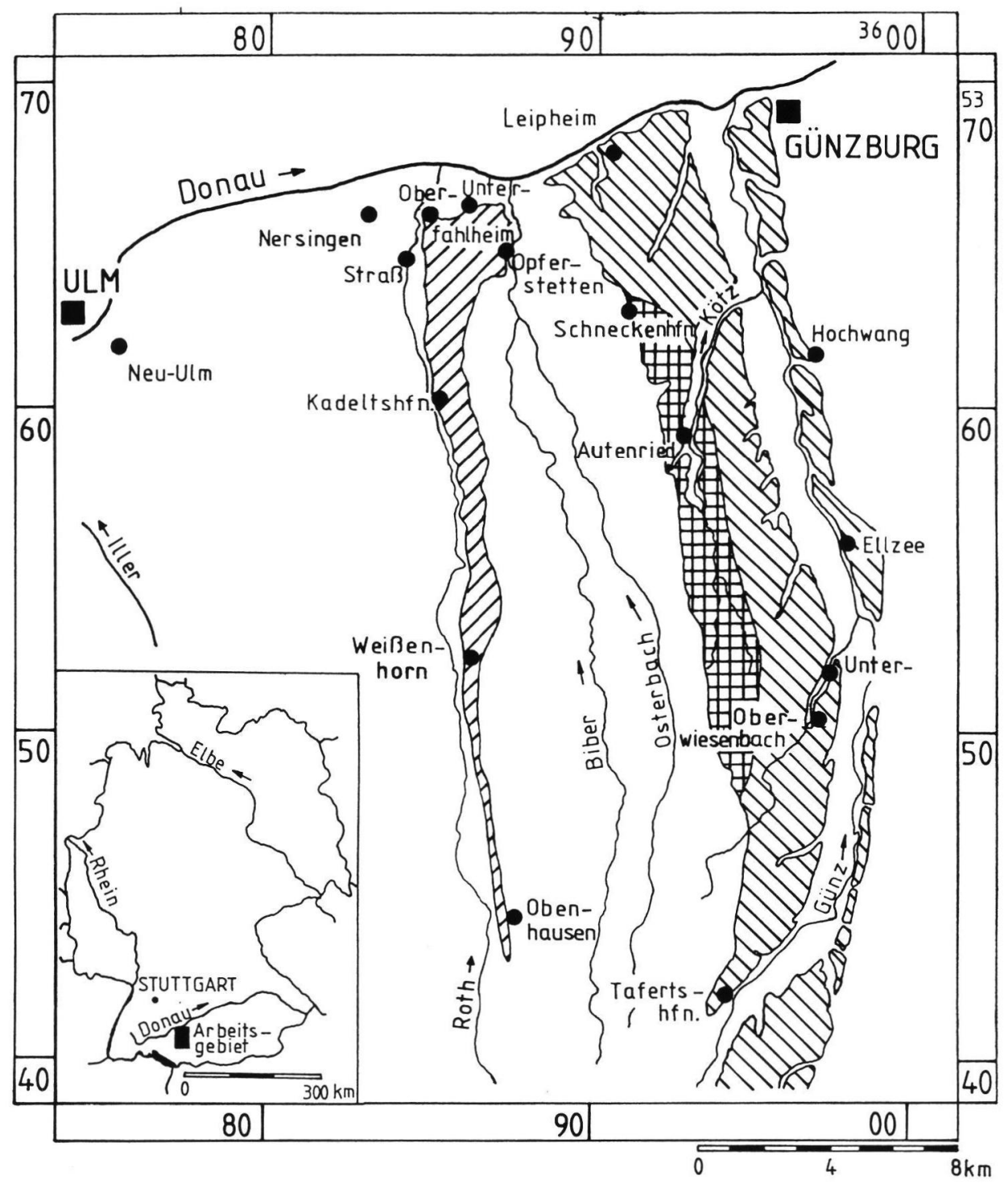

\section{LEGENDE}

Hochterrassenschotter; RiB

mit LöB- u. LöBlehmüberdeckung (ca. $6 \mathrm{~m}$ )

(nach JERZ et al. 1975) 
tierungen zeigen, daß im Westen der Günztal-Hochterrasse ein älteres glazifluviales Sediment erhalten geblieben ist.

Die Löß-Deckschicht in Autenried ist, wie schon LöSCHER 1976, LÖSCHER et al. 1979 und RÖGNER et al. 1988 festgestellt haben, in eine Würm- und eine Rißlößablagerung zu gliedern (vgl. Kap. 3.2). Demnach entstand der Schotterverwitterungslehm (2. fossiler Boden, Abb. 4) im liegenden Schotter mindestens im Mindel/Riß-Interglazial. Die Ablagerung des Schotters selbst fällt somit mindestens in die Mindeleiszeit. Diese Aussage wird durch die Verbindung der Schotterbasis des UnterwiesenbachSchneckenhofener Schotters mit der des mindeleiszeitlichen Grönenbach-Schwaighausener Schotters (=mindeleiszeitlicher Gletscherabfluß aus der Typregion PENCKS) bekräftigt.

Demgegenüber führt die Untersuchung des Deckschichtenprofils am Günzburger Krankenhaus zu dem Ergebnis, daß der Tafertshofen-Günzburger Schotter in der Rißeiszeit abgelagert wurde.

Die genauere zeitliche Einstufung dieser oberen Hochterrasse (oHT) mittels des Deckschichtenprofils Günzburg in eine ältere Phase der Rißeiszeit kann auch durch Vergleiche mit dem ,jungrißzeitlichen" Steinheimer Schotter (UHT) sowie dessen Deckschichten erfolgen, die deutlich anders gegliedert sind (Rögner et al. 1988). Im Profil Günzburg befindet sich der 1 . fossile Boden im unteren Abschnitt der Löß-Deckschicht; darunter folgt ein Schotterverwitterungslehm. Im Profil Steinheim hingegen liegt der 1. fossile Boden im Hochterrassenschotter selbst (vgl. Kap. 3.4 und RÖGNER et al. 1988: 63-65). Diese unterschiedliche Position des 1. fossilen Bodens auf der oberen und unteren Hochterrasse führt $\mathrm{zu}$ folgender stratigraphischchronologischer Interpretation der Schotterkörper: Im älteren Riß (Hauptriß i. S. Grauls 1962) lagerten die Schmelzwässer des Illergletschers die obere Hochterrasse im Günztal ab. Die besondere fluviale Dynamik dieses Schotterstroms führte bei Ellzee (siehe Kap. 3.3) dazu, daß der ältere, d. h. mindeleiszeitliche Schotter (der UnterwiesenbachSchneckenhofener Schotter) auf der Westseite des unteren Günztals erhalten geblieben ist.

Unterbrochen durch ein Interglazial (Beleg $=$ Schotterverwitterungslehm im Profil Günzburg, Abb. 6, Bvt: ab $8,3 \mathrm{~m}$ ), wird im jüngeren Riß (Jungriß i. S. Grauls 1962) die untere Hochterrasse im bayerischen Rothtal abgelagert. Gleichzeitig wird Löß auf den oberen Hochterrassenschotter im Günztal sedimentiert. Nach dem Abschmelzen des Illergletschers erfolgte im Eem auf der oberen Hochterrasse eine Bodenbildung im zuvor sedimentierten Rißlöß (Abb. 6, 1. fSBvt), während auf der unteren Hochterrasse sich dieser Boden im Hochterrassenschotter als Verwitterungslehm entwickelte (siehe RöGNER et al. 1988: 64, Fig. 6).
Die Deckschichtenuntersuchungen im unteren Günztal haben ergeben, daß entgegen LÖsCHER (1972, 1976) und LösCHER et al. (1979) glazifluviale Sedimente im unteren Günztal sowohl in der Mindeleiszeit (Westen) als auch in der Rißeiszeit (Osten) abgelagert wurden. Die flächenhafte Kartierung der verschiedenen Löß-Deckschichten mittels des Peilstangenbohrgerätes erlaubte die Abgrenzung eines mindestens mindeleiszeitlichen und eines rißeiszeitlichen Schotters (siehe Abb. 8).

\section{Danksagung}

Der deutschen Forschungsgemeinschaft danken wir für die Sachmittelzuwendungen, ohne die die Geländearbeiten und die TL-Datierungen nicht hätten durchgeführt werden können. Der Leitung des Instituts für Bodenkunde der Landes-, Lehr- und Versuchsanstalt für Landwirtschaft, Weinbau und Gartenbau in Trier sei dafür gedankt, daß S. Miara die gesamten Laboruntersuchungen in den dortigen Laboratorien durchführen konnte.

\section{Schriftenverzeichnis}

Bente, B. \& Löscher, M. (1987): Sedimentologische und stratigraphische Untersuchungen an Lössen südlich Heidelberg. - Göttinger geogr. Abh., 84: 9-17, 4 Abb.; Göttingen

- \& SCHWEIzER, V. (1988): Zur Korngrößenverteilung in Lößprofilen aus dem westlichen Kraichgau (Baden Württemberg). - Heidelberger geowiss. Abh. 20: 5-19, 4 Abb., 2 Tab.; Heidelberg

Bibus, E. (1974): Abtragungs- und Bodenbildungsphasen im Rißlöß. - Eiszeitalter u. Gegenwart, 25: 166-182, 6 Abb.; Öhringen (Rau)

Bludau, W,. (1995): Pollenanalytische Untersuchungen an interglazialen Sedimenten vom Füramoos (Blatt 8025 Bad Wurzach/Oberschwaben) mit Anmerkungen zur Gliederung des Frühwürms. - Abh. Geol. L.-Amt Baden-Württ.; Freiburg i. Breisgau (im Druck)

BRUNNACKER, K. (1957): Das Lößprofil von Kitzingen (Unterfranken). Ein Beitrag zur Chronologie des Paläolithikums. - Germania, 34: 3-11, 3 Abb., 1 Tab.; Berlin - (1958): Zur Parallelisierung des Jungpleistozäns in den Periglazialgebieten Bayerns und seiner östlichen Nachbarländer. - Geol. Jb., 76: 129-150, 5 Abb., 3 Tab.; Hannover

EBerL, B. (1930): Die Eiszeitenfolge im nördlichen Alpenvorlande. - VIII + 427 S., 10 Abb., 2 Taf., 1 Kt.; Augsburg (Filser)

Graul, H. (1952): Zur Gliederung der mittelpleistozänen Ablagerungen in Oberschwaben. - Eiszeitalter u. Gegenwart, 2: 133-146, 4 Abb.; Öhringen (Rau)

- (1953): Zur Gliederung der Würmeiszeit im Illergebiet. - Geologica Bavarica, 18: 13-48, 1 Profiltaf., 3 Abb.,; München

- (1962): Eine Revision der pleistozänen Stratigraphie des schwäbischen Alpenvorlandes. - Peterm. geogr Mitt., 106: 253-271, 8 Fig.; Gotha

- (1968): Führer zur zweitägigen Exkursion im nördlichen Rheingletschergebiet August 1968. - Heidelberger geogr. Arb., 20: 31-78, 6 Fig., 7 Kt., 2 Tab.; Heidelberg 
- Schaefer, I. \& Weidenbach, F. (1951): Quartärgeologische Exkursion durch die Riß-Lechplatte. - Geologica Bavarica, 6: 91-117, 9 Abb.; München

Habbe, K. A. \& Rögner, K. (1989a): Bavarian Alpine Foreland between rivers Iller and Lech. - Geoöko-Forum, 1: 181-222, 30 Fig., 6 Tab.; Darmstadt

- (1989b): The Pleistocene Iller glaciers and their outwash fields. - Catena suppl., 15: 311-328, 4 Abb.; Cremlingen

HÄDRICH, F. (1970): Zur Anwendbarkeit einiger bodenkundlicher Untersuchungsmethoden in der paläopedologischen und quartärgeologischen Forschung unter besonderer Berücksichtigung der Untersuchung von Proben aus Lößaufschlüssen. - Ber. Naturf. Ges. Freiburg i. Br., 60: 103-137, 3 Abb., 6 Tab.; Freiburg i. Breisgau

Jerz, H.; Stephan, W.; Streit, R. \& Weinig, H. (1975): Erläuterungen zur Geologischen Übersichtskarte des IllerMindel-Gebietes 1:100000. - Bayer. Geol. L.-Amt, 37 S., 1 Beil., München

- \& WAGner, R. (1978) mit Beitr. von Bader, K.; SALger, M.; Schuch, M. \& Weinig, H.: Erläuterungen zu Blatt 7927 Amendingen. - Geol. Kt. Bayern, 1:25000, 20 Abb., 7 Tab., 4 Beil., München

- \& Doppler, G. (1990), mit Beitr. von Roppelt, Th. \& Zöller, L.: 9. Tagung des Arbeitskreises Paläoböden der Deutschen Bodenkundlichen Gesellschaft. Paläoböden in Bayerisch Schwaben, Günzburg, 24.26.5.1990. - Bayer. Geol. L.-Amt, 30 S.; München

LÉger, M. (1987): Micromorphologie de Loess et Paleosols intraloessique en Souabe et Baviere. - Micromorphologie des Sols - Soil Micromorphology, 611-618, 10 Fig., 1 Tab.; Paris

LieBeroth, I. (1959): Beobachtungen im nordsächsischen Lößgebiet. - Z. Pflanzenernähr., Düngung, Bodenkde., 86: 141-155, 4 Abb., 2 Tab.; Weinheim

Löscher, M. (1972): Probleme der Quartärgeologie in der nördlichen Iller-Lech-Platte. - Führer 16. wiss. Tagung DEUQUA 1972 Stuttgart-Hohenheim, 22-25; Stuttgart - (1976): Die präwürmzeitlichen Schotterablagerungen in der nördlichen Iller-Lech-Platte. - Heidelberger geogr. Arb., 45, XIV + 157 S., 26 Abb.; 4 Tab., 8 Taf., 11 Prof., 4 Kt.; Heidelberg

- \& LÉGer, M. (1974): Probleme der Pleistozänstratigraphie in der nördlichen Iller-Lech-Platte. - Heidelberger geogr. Arb., 40: 59-76, 1 Kt., 4 Fig, (Hans GraulFestschrift); Heidelberg

- Schies, A.; Léger, M. \& Dabelstein, H.-J. (1979): Pedologische Untersuchungen in den Deckschichten des oberen Hochterrassenschotters im Günztal und ihre Aussagen für die Schotterstratigraphie in der Iller-LechPlatte. - In: Graul, H. \& LÖsCHER, M. (Hrsg.): Sammlung quartärmorphologischer Studien II, Heidelberger geogr. Arb., 49: 179-193, 1 Kt., 5 Fig.; Heidelberg

Miara, S.; ZÖller, L.; RÖGNer, K. \& Rousseau, D.-D. (1996): Quartäraufschlüsse bei Baltringen/Riß und Gliederung des Riß-Komplexes - neue stratigraphische, pedologische und geochronologische Aspekte. - Z. Geomorph. N. F., 40, 2: 209-226, 5 Fig., 1 Tab.; Berlin, Stuttgart.

PÉcsi, M. (1991): Problems of Loess Chronology. - GeoJournal, 24.2: 143-150, 2 Tab.; 2 Fig.; Dordrecht

PenCK, A. \& BRÜCKNER, E. (1901-09): Die Alpen im Eiszeitalter. - 3 Bdc., 1199 S., 88 Abb., 1 Taf., 5 Kt.; Leipzig (Tauchnitz)

Rohdenburg, H. \& Meyer, B. (1979): Zur Feinstratigraphie und Paläopedologie des Jungpleistozäns nach Untersuchungen an Südniedersächsischen und Nordhessischen Lössprofilen. - Landschaftsgenese u. Landschaftsökologie, 3: 1-89, 25 Abb., 12 Tab.; Braunschweig

RÖGneR, K.; Löscher, M. \& ZÖLler, L. (1988): Stratigraphie, Paläogeographie und erste Thermolumineszenzdatierungen in der westlichen Iller-Lech-Platte (Nördliches Alpenvorland, Deutschland). - Z. Geomorph., N. F., Suppl.-Bd. 70: 51-73, 9 Fig., 5 Tab.; Berlin, Stuttgart

Semmel, A. (1968): Studium über den Verlauf jungpleistozäner Formung in Hessen. - Frankfurter geogr. H., 45 , 133 S., 35 Abb., 2 Tab.; Frankfurt a. Main

- (1969): Bemerkungen zur Würmlößgliederung im Rhein-Main-Gebiet. - Notizbl. hess. L.-Amt Bodenforsch., 97: 395-399, 1 Abb.; Wiesbaden

SinN, P. (1972): Zur Stratigraphie und Paläogeographie des Präwürm im mittleren und südlichen IllergletscherVorland. - Heidelberger geogr. Arb., 37, XVI + 159 S., 21 Abb., 11 Tab., 13 Taf., 12 Prof., 5 Kt.; Heidelberg

Schönhals, E.; Rohdenburg, H. \& Semmel, A. (1964): Ergebnisse neuerer Untersuchungen zur Würmlöß-Gliederung in Hessen. - Eiszeitalter u. Gegenwart, 15: 199206, 1 Abb.; Öhringen (Rau)

SChwertmann, U. (1964): Differenzierung der Eisenoxide des Bodens durch Extraktion mit AmmoniumoxalatLösung. - Z. Pflanzenernähr., Düngung, Bodenkde., 105, H. 3: 194-202, 4 Tab.; Weinheim

Turon, J.-L. (1984): Direct Land/Sea correlations in the last interglacial complex. - Nature, 6/1984, Vol. 309: 673676, 2 Fig.; London

Van Vliet-Lanoe, B. (1989): Dynamics and extent of the weichselian permafrost in western Europe (Substage 5E to stage 1). - Quaternary International, Vol. 3/4: 109-113, 2 Fig.; Oxford

ZÖLLER, L. (1989): Geomorphologische und geologische Interpretation von Thermolumineszenz-Daten. - Bayreuther geowiss. Arb., 14: 103-112, 1 Abb., 1 Tab.; Bayreuth

- Stremme, H. \& Wagner, G. A. (1988): Thermolumineszenz-Datierung an Löss-Paläoboden-Sequenzen von Nieder-, Mittel- und Oberrhein/Bundesrepublik Deutschland. - Chemical Geology (Isotope Geoscience Section), 73: 39-62, 3 Tab., 11 Abb.; Amsterdam

Manuskript eingegangen am 24.01.1995 